\title{
Implementation of Navigation System using Li-Fi Technology
}

\author{
N A Pande, Jayant Kshirsagar
}

\begin{abstract}
In This Paper, an Indoor navigation system is proposed for persons with visually disability that utilize VLC (Visible Light Communication) Technology. Using VLC we can provide precise locational coordinates or directions regarding their journey. Light Fidelity (Li-Fi) refers to the VLC technology. VLC is based on the concept that uses visible light as a means of communication enabling faster flow of information [1]. The principle of VLC is that it uses light as a medium to deliver high speed communication. The main idea is to create internal navigation system for the area to create automatic navigation for the visitor who are visually challenged. The Li-Fi is preferred over Wi-Fi because Wi-Fi is nothing but radio-telecommunication which effective within premises, while Li-Fi is effective for faster communication in restricted places without any hurdles. The concept of Li-Fi is used to give the navigation path for visually challenged person and particularly voice guidance of the walk way. Headphone is used to give the voice message to such people. The data is transmitted through the LED light which emits the visible light and an embedded system accepts from receiver. The received data is converted into audio format for the blind people. The suitability of system has been confirmed by means of useful prototype through a trail with blind person.
\end{abstract}

Keywords : VLC, Li-Fi, High speed data transmission

\section{INTRODUCTION}

Visually challanged people, either partially or toal blind, always struggle at public places like Bus stations, Railway Terminals, Hospitals, Malls etc. By design, such places are not equipped to take special care of such visually challened people. Several "everyday" objects can cause serious accidents at such places. In order to aviod this great difficulty, Visible light communication (VLC) is used. VLC enables flow or transfer of information and data using the visible light[1]. This can help the visually challenged people to navigate at certain indoor places so that they can move without depending on anybody within such areas. So, the main purpose of the VLC ecosystem is to give real time, accurate inputs and information on location and directions to its users (i.e. visually challanged people) so that they can reach their destination smoothly using the shortest path in an indoor space. In this era where wireless technology is widely developing, the wifi is practically used for all general purposes where wireless network is needed, whether in households, buildings or commercial spaces. Whereas, Li-Fi

Revised Manuscript Received on May 15, 2020.

* Correspondence Author

Mrs. N A Pande*, Assistant Professor, YCCE Nagpur

Jayant Kshirsagar, System Engineer at TCS, Nagpur

(C) The Authors. Published by Blue Eyes Intelligence Engineering and Sciences Publication (BEIESP). This is an open access article under the CC BY-NC-ND license (http://creativecommons.org/licenses/by-nc-nd/4.0/) is suitable for faster communication in restricated area without any obstacles[2]. Li-Fi is easy to implement since it uses visible light as means of transfer of information and visible light is there every where. While Wi-Fi uses radio-frequency for transfer of information, Li-Fi uses visible light for the same purpose. However, it is superior over Wi-Fi. It offers a wider bandwidth channel for data transmission and higher data transfer speeds. Also, it can be safely used in some restricted areas like aircraft cabins, Military areas and Hospitals which are sensitive for electromagnetic interference. The most important feature of $\mathrm{Li}-\mathrm{Fi}$ is that transfer of data is possible through LED. It can be used securly at indoor locations where enough light would be available. Li-Fi works through flickring of the LED in such a way that if LED is ON, user can send a digit of 1 , if it is OFF then user can send a string of 0 , and it create binary code of 1 's and 0 's with a LED flickring which is not visible for human eye. Hence the data transmitted by LED light is received by the electronic circuit with photodiode. This means that this process gives light as well as wireless data trasmission. Since the Li-Fi does away with the electro magnetic or radio frequency waves, it is not only safer but alos a cheaper and faster alternative to Wi-Fi. Prof Harald Haas is said to have articulated the word Li-Fi for the first-time at his TED Global Talk in 2011 at Edinburgh, where he put forth the notion of this wireless technology for data transmission using light source. Since then, several research associations, industries and organizations have worked on this novel concept to experiment and enhance this technology. According to available information, the data transmission speed possible using VLC techonology is more than 10 Gbps. In implementation of Li-Fi technology, the normal Light Emitting Diod bulbs are utilized which are applied a constant supply of current. It is possible to produce high speed by fast variation of the electric supply. By flashing the LED several time the intended data is delivered. When LED is ON the microchip can convert the specific information in the form of light-beam. This light is transmitted by the light emitting diode and is received a photo-detector. The photo-detector is an extremely light responsive instrument which trasforms the signal to its original data. As the flashing rate is extremely fast, the end result of light emitting diode would appear consistent to the human optics. In the Li-Fi technology, the information transmission speed can be enhanced by multiple side by side data transfer using LED grouping or such arrangement. In this case, each light emighting diode constituent of the bulb can carry or broadcast a separate or distinct flow of information.

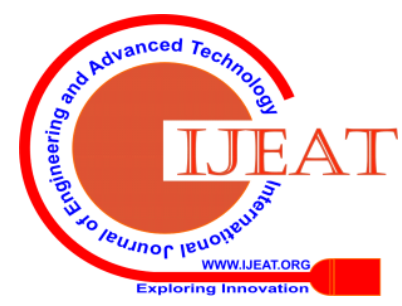




\section{Implementation of Navigation System using Li-Fi Technology}

\section{METHODOLOGY}

Although a constant electric flow is applied to the LED, it is possible to change the resultant ouflow by constantly flickring the light at exceedingly high pace. The Li-Fi assembly uses this notion of possible variance in output during the data transfer.

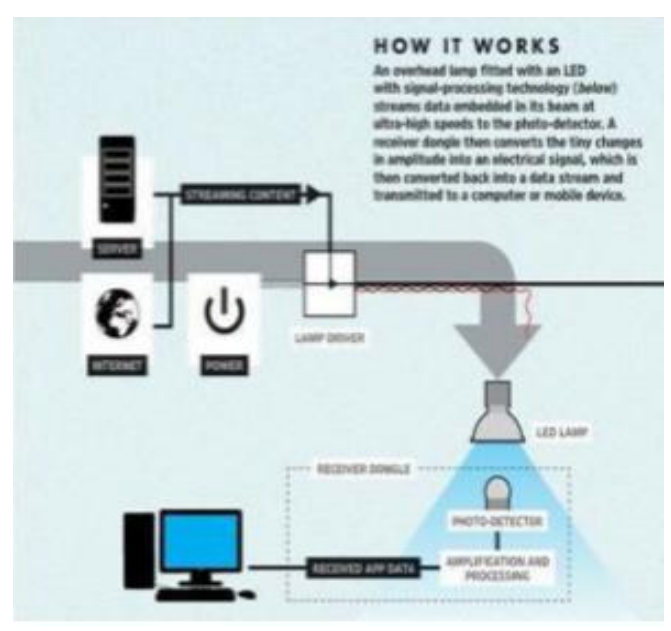

Figure1: Flow of data transfer using Li-Fi

As shown in figure $1 \& 2$, the microchip in the LED will do the processing of the information which is embedded in the light beam. The lamp driver at the input end ensures that the data is flown as a continuous current. When LED is ON the chip converts digital data in form of light. On the receiver end, a receiver dongle containing the photo-dectector receives the light and processes the same by converting the minute variances in the amplitude into an electric-signal. If the LED is $\mathrm{ON}$, it sends a digit 1 , if it is OFF it sends digit 0 . By continously sending data, the receiver detects the original message. This electric-signal is then transformed back into the original data flow which is detected in the form of audio format. Li-Fi technology uses semiconductor device LED. The continuous and fast illumination of the LED produces binary codes which can continuously send this information flow by very small variance of displacement in the wave form. The main important unit of the Li-Fi system is its high luminosity LED bulb which works as a source and other is silicon photo-diode or detector which reacts as a receiver .

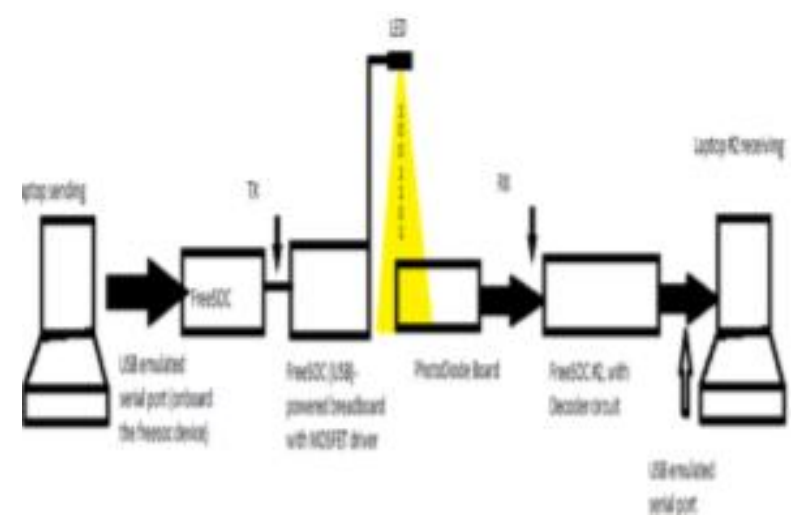

Figure2: Data Transmission and Receiving

The user can recognize it's position within the range of 1 to 2 kms by visible light communication. In addition, the VLC technology makes it possible for the user to figure out on which floor they are located.

\section{DESIGN AND IMPLEMENTATION}

The system comprises of LED bulb, a receiver and headphone. The LED transmit visible light ID using visible light communication. It is then received by the receiver. The resultant message is combined to audio and sent to headphone via text to audio converter. The text to audio converter system is as shown below. The intensity or brightness of a LED is normally correlated to the input electricity flow - this is the basic idea in its usage. Consequently, the luminosity of the LED corresponds to the speed of information flow. The micro-controller ports are not well equipped to handle the incremental flow of electricity to provide higher luminosity required for faster information flow. To overcome this difficulty, a motor driver IC and transistor is used as a switch, which made it possible to switch a larger current faster. The receiver changes the incoming light into electrical signal using a photo-diode. The receivers need to change the current to voltage in system to amplify and measure it. Distance between the source or LED and the receiver cannot be constant as the receiver would move according to inputs being received from the source. Depending on the distance between them, the signal frequency could vary from very low to very high. In order to eliminate this variance, an automatic gain controller can be used. In lieu of that, a variable resistor is used here. This device increases or lowers the input voltage to a selected output voltage.

The major hardware tools used are as shown below.

1. Text to audio converter - it is used for conversion of text output signal to audio signal

2. Micro-controllers - used for controlling operations of different peripherals

\subsection{Block diagram of transmitter}

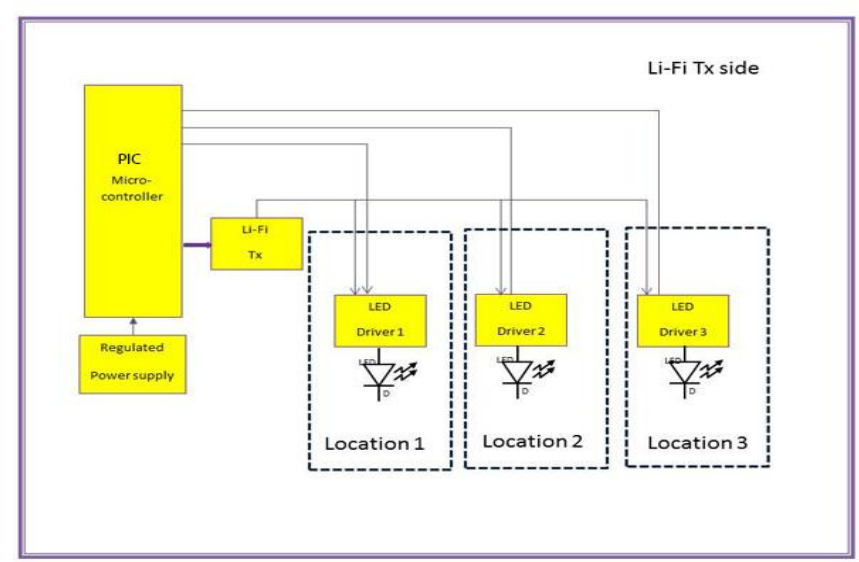

Figure3. Transmitter

\subsection{Block diagram of receiver}

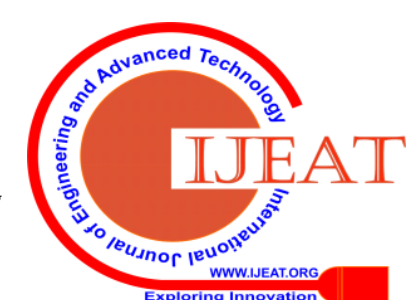




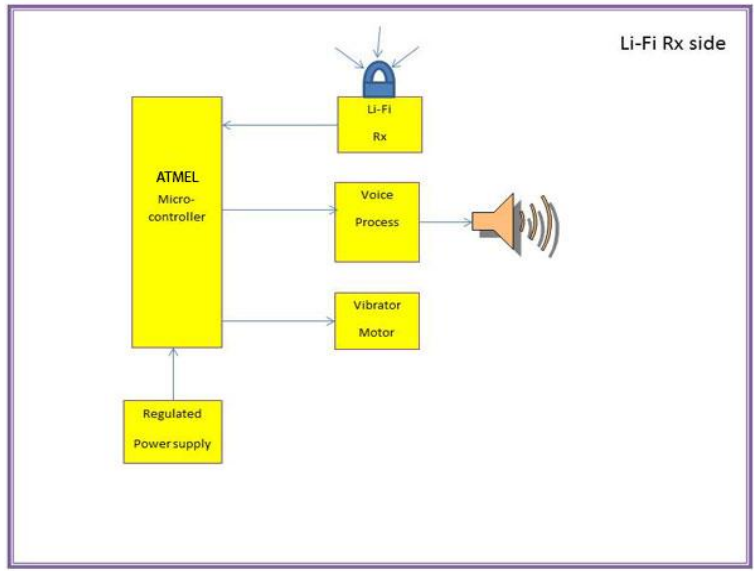

Figure 4. Receiver

For implementation of Li-Fi technology, LED (Light Emmiting Diode) i.e. light bulb can be turned ON and OFF frequently to generate signals. An appropriate light receiver is constructed for receiving the LED signals. The flow of information can be controlled by varying the luminosity of light. Li-Fi technology ensures that the light bulb is continuously switching ON and OFF to millions of time a second. This is beyond the cotemplation of the human eye to notice the changes or flickring of light. The digital string of 1 is transferred when the LED is ON. The digital string of 0 is transferred when the LED is OFF. The LED can be turned ON and OFF enabling instant data transmission. The data to be transmitted is fed to LED light bulb which is then sent to photodiode at rapid speed. The receiver can transform the smallest of variance in quick illumination of LED bulb into electric current. The electric signal is then turned back in to binary flow of information.

The transmission module is fitted in walls and ceiling . It transmits the modulated information via LED . the receiver module demodulates the binary encoded data . The output response is received in the form of voice signal to the receiving person. The receiver module includes photo transistor. The information is received by the photo resistor from the LED's connected in transmitter module. The information consists of navigating directions and location. Whenever the receiver module comes in the range of transmission area the respective location message is sent to receiver. The information is also stored in IC ATMEGA328P which is further used to initiate the sunrom TTS (Text To Speech) module. This provides assistance to the visually challanged people at designated indoor places. The ON and OFF pattern is generated by the transmission module for the LED's. The photodiode in receiver module detect this ON and OFF pattern of LED's. It captures the $\mathrm{ON}$ and $\mathrm{OFF}$ sequence and further generates the corresponding binary algorithm of the received input.

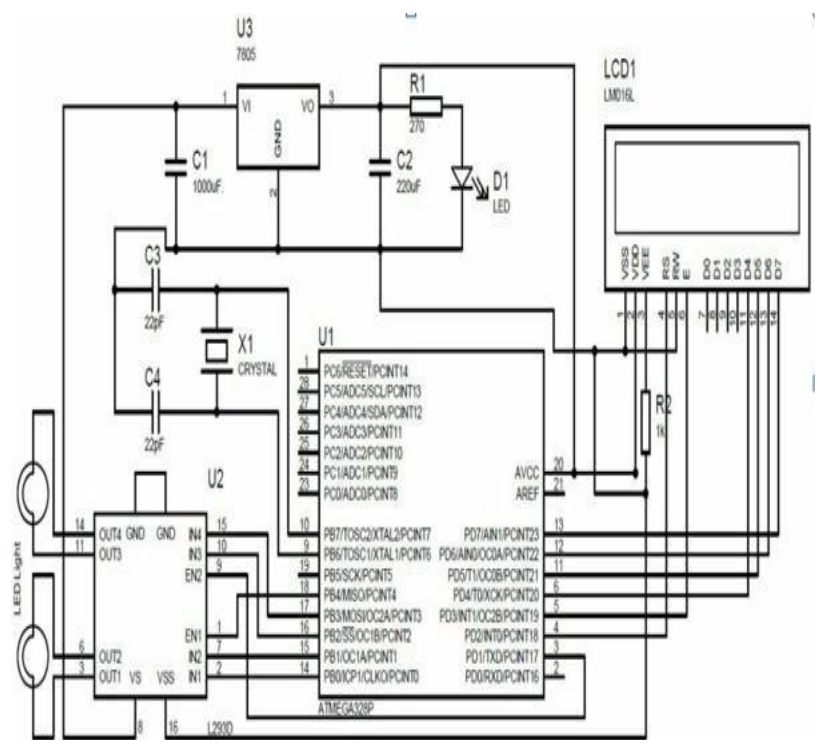

Figure 5 Transmitter circuit

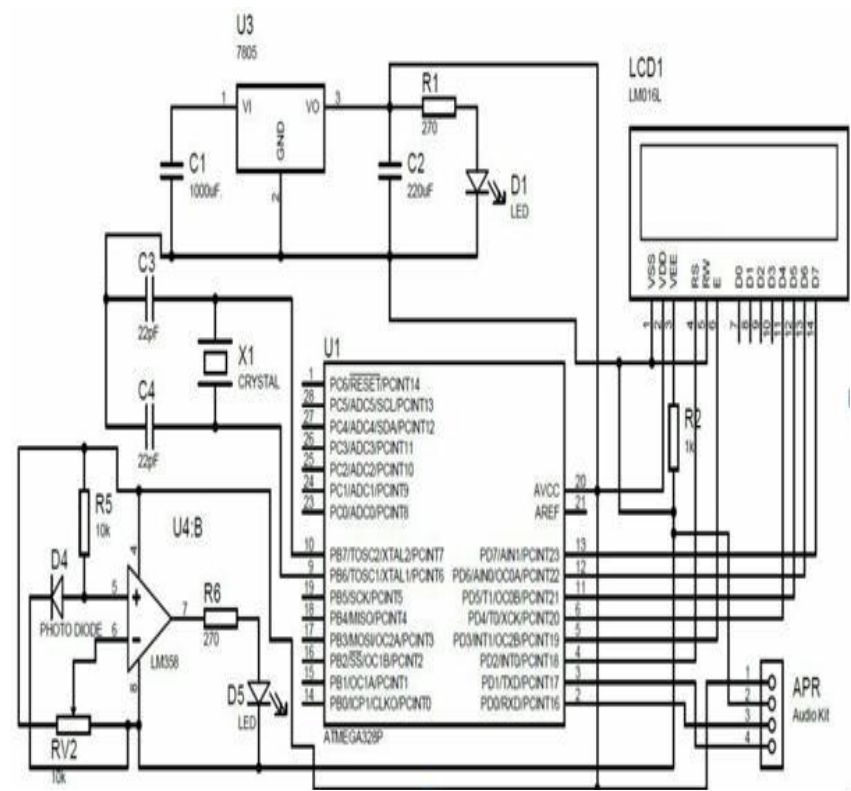

Figure 6 Receiver circuit

The receiver module converts incident light into current using a photo diode. The Aurdino does not receive a voltage more than $5 \mathrm{~V}$. Hence processing of pulse signal is done between the photo diode and Aurdino in the receiver module for its correct interpretation. The current signal is converted into voltage signal to amplify and compare it. This has been don keeping in mind the possible changes in distance and location between the source and receiver. An automated gain controller is designed to neglect too high or too low signals. The function of this component is to increase or lower the input voltage to desired output voltage.

\section{RESUlT:}

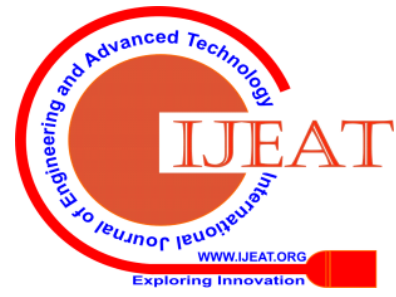




\section{Implementation of Navigation System using Li-Fi Technology}

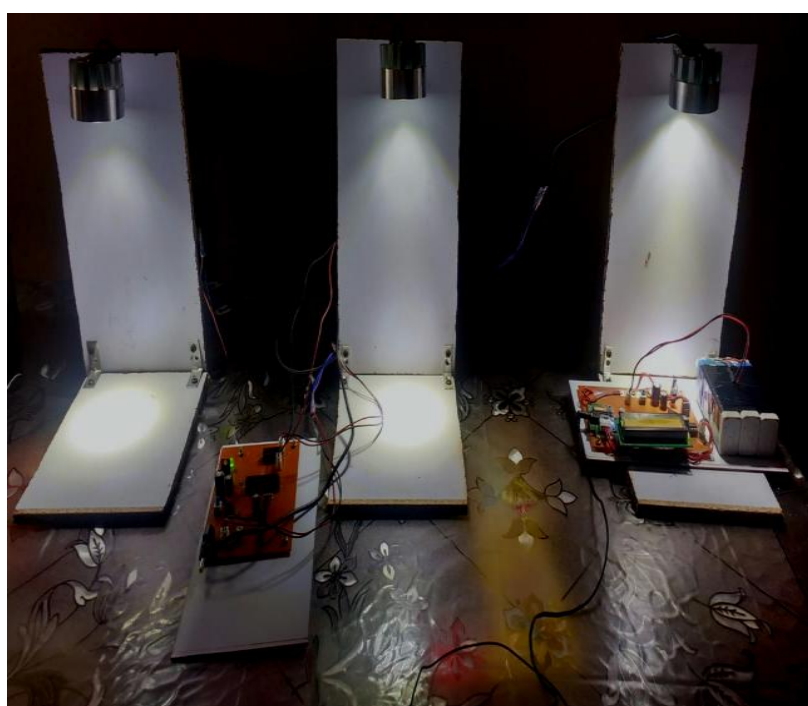

Figure 7 Implemented System

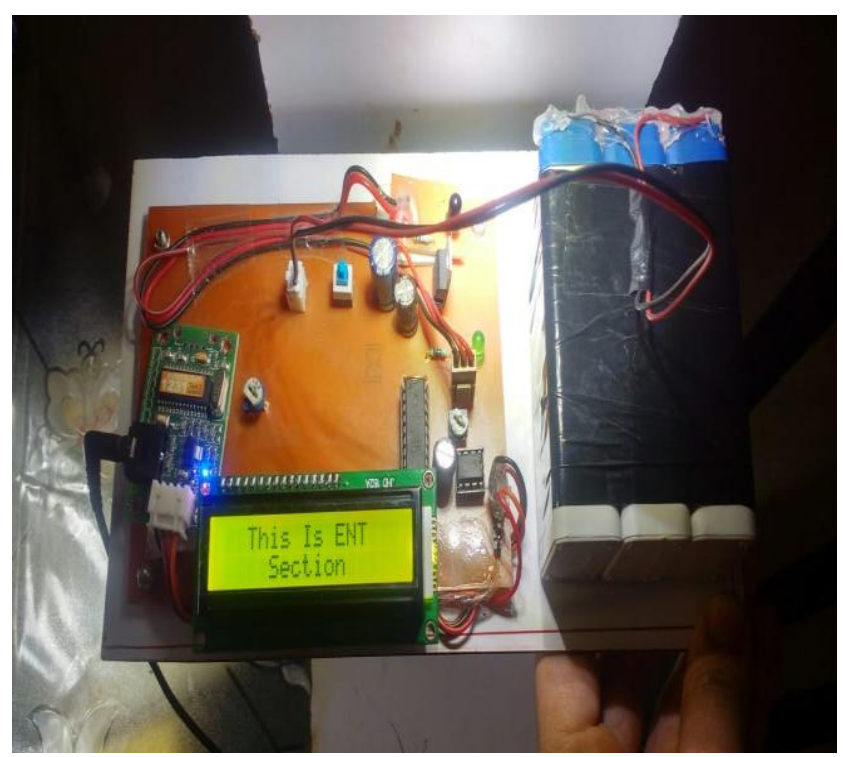

Figure 8 Result

Data transmission by the virtue of visible light communication using LED and photo diode is implemented. We have used LED bulbs for transmission of data and a photo diode for data reception.

\section{CONCLUSION:}

Transmission and reception of data at very high rates is possible using Li-Fi technology by simply switching $\mathrm{ON}$ and OFF the LED's. With the increase in light intensity the data transmission rate increases. Thus the transmission and reception of data is completely depends on light. In the absence of light we cannot establish communication between two devices. $\mathrm{Li}-\mathrm{Fi}$ is executed for the purpose of transmitting data with higher bandwidth and higher range. The use of radio waves that are harmful to us is reduced. $\mathrm{Li}-\mathrm{Fi}$ is complimentary wireless communication system. $\mathrm{Li}-\mathrm{Fi}$ is not replacement of $\mathrm{Wi}-\mathrm{Fi}$. If $\mathrm{Li}-\mathrm{Fi}$ technology is practically implemented with each LED bulb can be used similar to a Wi-Fi hotspots leading to a clean, green and safe future.

\section{REFERENCES}

1. R. Mahendran, "Integrated LiFi(Light Fidelity) for smart communication through illumination”, IEEE explore January 2017,

2. Harald Haas ; Liang Yin ; Yunlu Wang; Cheng Chen,"What is LiFi?", Journal of Lightwave Technology Volume: 34 , Issue: 6 , March15, 152016 , Page(s): 1533 - 1544

3. H.Haasand N. Serafimovski, "LiFi unlocking unprecedented wireless pathways for

4. our digital future," IEEE Com Soc Technology News, Dec.2016.

5. H. Elgala, R.Mesleh, and H. Haas, Indooroptical wireless communication :

6. Potential and state-of the art," IEEE Communications Magazine, vol.49, no.9, pp.56- 62, 2011.

7. L. Grobeand A. Paraskevopoulos, "High-speed visible light communication systems," IEEE Communications Magazine, pp.60-66, Dec.2013.

8. NASA (2015). [Online]. Available https://blogs.nasa.gov/kennedy/2015/07/30/partnership-maylead-to-a dvanced-communication-technology/

9. Jyoti Rani, Prerna Chauhan, Ritika Tripathi, - Li-Fi (Light Fidelity) The future

10. technology In Wireless communication, International Journal of Applied Engineering Research, ISSN0973 -4562Vol.7 No.11(2012).

11. Vitthal S Saptasagare - Next of Wi-fi a Future Technology in Wireless

12. Networking Li-Fi Led Over Internet of Things, International Journal of Emerging

13. Research in Management \& Technology ISSN:2278-9359 (Volume-3, Issue-3)(2014).

14. International Journal of Emerging Technology and Advance Engineering Website: www.ijetae.com (ISSN2250-2459, ISO9001:2008 Certified Journal, Volume5, Issue10, October2015): Smart Trolley System for Automated Billing using RFID and ZIGBEE

15. www.lificonsortium.org/

\section{AUTHORS PROFILE}

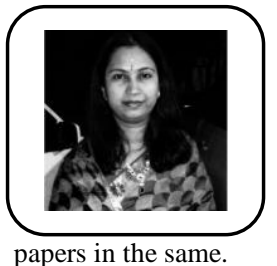

Nivedita A Pande, (M.Tech., Electronics)

Prof. Nivedita A Pande is the Assistant professor at department of Electronics and Telecommunication YCE, Nagpur. She has work experience of 12 yrs. She completed her post graduate from RTMNU university. Her area of research is an Embedded Systems. She has published more than 6 research

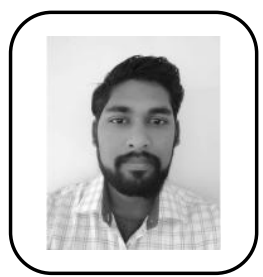

Jayant Kshirsagar (BE, Electronics \& Telecommunication)

Currently, Jayant Kshirsagar is working as an Middleware Developer in TATA consultancy services (TCS) BFSI Domain, Nagpur. He completed his graduation from

YCCE, Nagpur. He is expertise in Integration and API development.

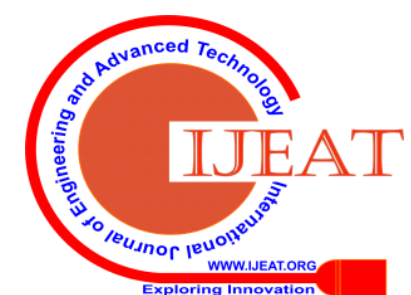

